\title{
The association between sleep and cognitive abnormalities in bipolar disorder
}

Bradley $\mathrm{AJ}^{1,2}$, Anderson $\mathrm{KN}^{4}$, Gallagher $\mathrm{P}^{1}$, McAllister-Williams $\mathrm{RH}^{1,3 *}$.

1. Institute of Neuroscience, Newcastle University, Wolfson Research Centre, Campus for Aging and Vitality, Westgate Road, Newcastle Upon Tyne, NE4 5LP

2. Eli Lilly and Company Limited, Lilly House, Priestly Road, Basingstoke RG24 9NL.

3. Northumberland, Tyne and Wear NHS Foundation Trust, Newcastle, UK

4. Regional Sleep Service, Freeman Hospital, Freeman Road, High Heaton, Newcastle upon Tyne NE7 7DN.

*corresponding author. Email hamish.mcallister-williams@ncl.ac.uk

\section{Acknowledgements and Financial Support}

We would like to thank all of the participants for taking part in the study. The study was sponsored by Northumberland, Tyne and Wear NHS Foundation Trust. Some funding to cover running costs was received from Eli Lilly as an unrestricted investigator initiated and led award. The design, conduct and analysis of the study was entirely under the control of the authors.

Word count (excluding references, tables and figure legends): 4616 Abstract word count: 246

Tables: 2

Figures: 3

References: 41 


\section{Abstract}

\section{Background}

Bipolar disorder is associated with attentional and processing abnormalities. Such abnormalities are also seen in healthy subjects with sleep disruption. We hypothesised cognitive abnormalities in bipolar disorder patients would be worse in those with objectively verified sleep abnormalities.

\section{Methods}

$46 \mathrm{BD}$ patients and 42 controls had comprehensive sleep/circadian rhythm assessment over 21 days alongside mood questionnaires. Cognitive function was assessed with a range of tasks including psychomotor vigilance test (PVT), Attention Network Task (ANT) and Digit Symbol Substitution Test (DSST). Bipolar disorder participants with normal and abnormal sleep were compared to age and sex matched controls.

\section{Results}

BD patients had longer response times and made more lapses (responses $>500 \mathrm{~ms}$ ) than controls on the PVT (both $p<0.001)$. However, patients with normal sleep patterns did not differ from controls while those with sleep abnormalities did $(p<0.001)$. An identical pattern of effects were seen with the ANT response times, with the abnormality in bipolar abnormal sleepers related to the executive attentional network. Similarly, patients made fewer correct responses on the DSST compared to the controls $(p<0.001)$. Bipolar normal sleepers did not differ while those with abnormal sleep did $(p<0.001)$. All these differences were seen in bipolar abnormal sleepers who were euthymic $(p<0.01)$ and across the main abnormal sleep phenotypes.

\section{Conclusions}

We confirm impairment in attention and processing in bipolar disorder. Rather than sleep abnormalities exacerbating such dysfunction, the abnormalities were confined to bipolar abnormal sleepers, consistent with sleep disturbance being the main driver of the cognitive abnormalities. 


\section{Introduction}

Bipolar disorder (BD) is associated with a range of well described cognitive dysfunctions as detailed in an individual patient data meta-analysis of 2876 subjects, with deficits seen across all cognitive domains tested (Bourne et al. 2013). While cognitive dysfunction is modestly associated with a history of psychotic symptoms and mania, it is seen across all clinical subgroups (Bora 2018), including in patients when euthymic (Robinson et al. 2006; Thompson et al. 2005). There is little evidence of progression of dysfunction over time (Samame et al. 2014). The dysfunction may relate to underlying attentional and processing speed abnormalities (Gallagher et al. 2014). The attentional abnormalities include increased intra-individual variability (IIV) in response times in attentional tasks (Gallagher et al. 2015). Sleep disruption, a common feature of all stages of BD, including euthymia (Harvey et al. 2005), can also cause attentional abnormalities (Lim and Dinges 2008). Again this is associated with an increased IIV, most commonly reported as an increase of 'lapses', or slow responses, in attentional tasks. Primary sleep disorders, such as sleep apnoea, also impact on attention and alertness (Bucks et al. 2013) and are underdiagnosed in BD (Soreca et al. 2015). Prior studies assessing cognition in patients with BD have rarely objectively assessed sleep alongside cognition and at best have relied upon self-report (Giglio et al. 2010;Kanady et al. 2017). There is a relative lack of correlation between objectively assessed and subjectively reported sleep abnormalities in bipolar disorder (Bradley et al. 2017; Harvey, Schmidt, Scarna, Semler, \& Goodwin 2005). Therefore, there is an important lack of data describing the association between objectively measured sleep abnormalities and cognitive function in BD. Previous work from this group objectively assessed sleep and circadian rhythm in detail in a well characterised cohort of BD patients (Bradley, Webb-Mitchell, Hazu, Slater, Middleton, Gallagher, McAllister-Williams, \& Anderson 2017). This revealed a range of sleep problems including obstructive sleep apnoea, circadian rhythm disturbance, hypersomnia and insomnia, with many patients having evidence of more than one of these disturbances. These objectively measured sleep disturbances were associated with impaired psychosocial functioning and quality of life. The aim of this study was to 
assess the relationship between objectively assessed sleep and cognitive function in BD patients. Specifically this study has compared cognition in BD patients with and without abnormal sleep to age and sex matched controls. Our hypothesis was that BD patients would demonstrate evidence of cognitive dysfunction and that this would be worse in, though not exclusive to, those patients with objectively verified sleep abnormalities.

\section{Method}

\section{Participants}

The study was approved by the National Research Ethics Service Committee North East - Newcastle \& North Tyneside. Outpatients with BD type I or II, in any mood state, were recruited from a research database, patient support groups, and NHS services in the North East of England. Healthy controls, matched by age and gender, were recruited from Newcastle University, local volunteer databases and hospital staff and their families. All participants provided written informed consent before taking part in the research. Participants were aged 18 to 65 years and fluent in English. Exclusion criteria were: Any significant medical or neurological disorder that might interfere with sleep or cognition; current alcohol or substance misuse disorder (defined by DSM IV criteria (American Psychiatric Association 1994); current shift work and previous significant head injury. A BD diagnosis meeting DSM-IV criteria was confirmed using the Mini International Neuropsychological Interview (MINI)(Sheehan et al. 1998). Patients with BD were excluded if they had had any changes to their psychotropic medication in the previous 4 weeks. Exclusion criteria for controls were: personal or first degree relative history of a DSM IV Axis I disorder as determined by clinical history; prescribed psychotropic medications and any known sleep disorder. Additionally controls had to be psychiatrically well, confirmed by MINI interview; have a 17 item Hamilton Depression Rating Scale score (HAMD-17) (Hamilton 1967) score <7; a Young Mania Rating Scale (YMRS) (Young et al. 1978) score <5; a Pittsburgh sleep quality index (PSQI) (Buysse et al. 1989) score $<5$ and Epworth sleepiness scale (ESS) (Johns 1991) score $<10$. 


\section{Overall study design}

The study was cross-sectional, with participants assessed over a three week period.

\section{Psychiatric Symptoms and Sleep Assessments}

Participants were assessed on days 1 and 21 . A comprehensive battery of questionnaires and rating scales were used to assess psychiatric symptoms. These included the 17 item GRID-HAMD (Williams et al. 2008), Beck Depression Inventory (BDI) (Beck et al. 1961) and YMRS (Young, Biggs, Ziegler, \& Meyer 1978). All medications used by the patients in the BD group were recorded.

A single night of home partial polysomnography (Embla Systems, Bloomfield, USA) was used to screen for sleep apnoea. Respiratory events were scored according to the standard criteria of the American Association of Sleep Medicine (AASM) (Kushida et al. 2005). An AHI of $>5 / h r$ was considered abnormal and indicative of sleep apnoea. Severity was defined as mild (AHI 5-15), moderate (AHI 15-30) or severe (AHI >30). Participants also completed the Restless Legs Syndrome (RLS) Rating Scale to assess for the presence and severity of RLS (Walters et al. 2003). This scale includes 10 questions each scoring 0-4 with a score of 1-10 representing mild, 11-20 moderate, 2130 severe and 31-40 very severe RLS.

For objective assessment of sleep/wake cycle (circadian rhythm), subjects wore a triaxial wrist accelerometer (GENEActiv; Activinsights) on their non-dominant wrist for all 21 days of the study alongside completing a daily sleep log. The analysis of this data has been previously described (Bradley, Webb-Mitchell, Hazu, Slater, Middleton, Gallagher, McAllister-Williams, \& Anderson 2017). In brief, the accelerometer data was analysed using an open source R package sleep detection algorithm, GGIR, which has demonstrated a high sensitivity and specificity to detect periods of sleep (van Hees et al. 2015). Sleep onset time, sleep offset time, TST, time in bed (TIB), sleep efficiency (defined as TST/TIB) and mean 24 hour sleep duration (defined as nocturnal sleep plus daytime naps) were all derived. Correlation analysis was performed to check agreement between sleep logs 
and accelerometer derived sleep variables. The relative amplitude between day and night activity was calculated during the least active 5 hours (L5) and most active 10 hours (M10) according to previously published methods (Van Someren et al. 1999). Participants were then identified as normal sleepers (6-10 hours sleep within 24 hours with a regular sleep wake cycle), short sleepers ( $<6$ hours nocturnal sleep), long sleep (>10 hours sleep within 24 hours (Kaplan et al. 2011)) and circadian rhythm disturbance, including a delayed sleep phase (habitual sleep onset after 2am), advanced sleep phase, irregular sleep wake pattern (three to four periods of sleep but no consolidated overnight period) or non 24 hour pattern in keeping with the definitions within the International Classification of Sleep Disorder $-3^{\text {rd }}$ edition (ICSD-3) (American Academy of Sleep Medicine 2014). Patient reported sleep logs were used to assist in the interpretation of actigraphy data.

\section{Cognitive assessment battery}

Verbal IQ was assessed with the national Adult Reading Test (NART) (Nelson 1982) and then a range of tasks were administered to assess psychomotor speed, attention, and executive components of cognition. This comprised of the Psychomotor vigilance Test (PVT) (Dinges and Powell 1985), the Attention Network Test (ANT) (Fan et al. 2002) and the Digit Symbol Substitution Test (DSST) (Wechsler 1981). Participants completed two DSST's one at the beginning of the cognitive testing session and one at the end of the cognitive testing session to assess for any change in performance across the testing session. Reaction time (RT) data from the PVT and the ANT were fitted to an exGaussian distribution (a mathematical convolution of the Gaussian normal and exponential distribution). The ex-Gaussian has been sown to approximate well to empirical RT distributions (Schmiedek et al. 2007); this distribution model produces three parameters: $m u$ and sigma, the mean and standard deviation of the Gaussian component; and tau, which defines the exponential component and represents the 'slow-tail' of the distribution (Ratcliff 1979). To derive these measures, Ex-Gaussian probability density functions were fitted to the distribution of valid response 
times of each individual using the DISTRIB toolbox (Lacouture and Cousineau 2008) in MATLAB ${ }^{\circledR}$ v.R2010b (The MathWorks Inc. Natick, MA).

The Newcastle Spatial Working Memory test (Pariante et al. 2012), a test of verbal learning, TRAILS A and B and digit span test (Lezak et al. 2004) were also performed. Tests were performed at the same time of day for controls and BD patients at the end of the sleep assessment period.

\section{Statistical analysis}

All statistical analyses were performed using IBM SPSS statistical package version 22. Data is reported as means with standard deviations. Normality of distribution of data was tested using the Shapiro-Wilk test. Log10 or square root transformations were used where necessary to normalise the data. Parametric tests (e.g. t-test) were used unless the data remained non-normally distributed despite transformation when equivalent non-parametric tests (e.g. Mann Whitney $U$ test) were used. In the comparisons between controls and BD patients, age and NART IQ score were examined as possible confounders. If there was a significant correlation of the outcome measure under investigation and either of these variables, then ANCOVA was performed. Mood was not examined in this way due to this being significantly different between controls and BD patients. Rather subgroup analysis was performed taking advantage of those BD patients who were euthymic. A score of $<8$ on the BDI was used to define 'euthymia' (Keller 2003). Note that all bipolar patients met euthymia criteria (YMRS < 10) with regards to manic symptoms. Overall, there was a strong correlation between HAMD-17 and BDI scores $\left(r_{(s)(45)}=0.831, p<0.001\right)$. The BDI was used in preference to the HAMD-17 due to it containing only one sleep variable, rather than the 3 within the HAMD-17. A significance threshold of $p<0.05$ was used for all analyses. 


\section{Results}

\section{Participants}

The participants and their sleep phenotypes have previously been described (Bradley, WebbMitchell, Hazu, Slater, Middleton, Gallagher, McAllister-Williams, \& Anderson 2017). In this current analysis only participants with complete actigraphy and overnight sleep oximetry data sets were included in the analysis. Eighty two participants, 46 with BD (16 BD I and $30 \mathrm{BDII})$ and 36 controls, completed the study protocol. Table 1 shows the participant characteristics. Groups did not differ significantly with regard to age or gender but participants with BD had a greater body mass index (BMI) and scored more highly on mood rating scales. Twenty one participants with BD scored $\geq 8$ on the HAMD-17 (range 8 -35), but none of the patients were considered clinically to be in a manic or hypomanic episode for the duration of the study (YMRS range $0-10$ ). Of the 46 BD patients 28 had objectively defined abnormal sleep including obstructive sleep apnoea (OSA) ( $n=12)$, circadian rhythm sleep disorder (CRSD) $(n=12)$, long sleep $(n=14)$, short sleep $(n=4)$ or a combination of these abnormalities. Sixteen had normal objective measures of sleep with the absence of sleep apnoea confirmed with an overnight sleep study. Two BD participants with normal accelerometry defined sleep patterns did not complete the overnight sleep apnoea study so were not included in the normal sleeping group. On this basis, the BD patients were divided into those with objectively normal $(n=16)$ and objectively abnormal sleep $(n=28)$. The characteristics of the various bipolar subgroups is shown in Table 2. 36 controls had objectively verified normal sleep without any level of obstructive sleep apnoea and were included in the analysis. Two BD participants did not complete the full battery of cognitive tests, one who became anxious and asked to stop and the other for undisclosed reasons. One aged 55 years, was a normal sleeper and had a BDI score of 8 . The other aged 25 years, was a normal sleeper with a BDI of 28 . Their data is included for the tests they completed.

Table 1 and 2 near here 


\section{Psychomotor vigilance test (PVT)}

The PVT was completed by 36 controls and 46 BD patients. One BD patient was omitted from the analysis as the number of lapses committed was more than 3 times the interquartile range and they were deemed an extreme outlier. As anticipated, BD patients differed from control participants having longer mean response times (RTs: $360 \pm 50 \mathrm{~ms}$ vs $325 \pm 32 \mathrm{~ms} ; \mathrm{p}<0.001 ; \mathrm{g}=0.81,95 \% \mathrm{Cl}=0.35$ -1.26 ) and making more 'lapses' (RT > 500ms; $5.1 \pm 6.4$ vs $1.8 \pm 2.8 ; \mathrm{p}<0.001 ; \mathrm{g}=0.64,95 \% \mathrm{Cl}=$ $0.19-1.09)$. There were no missed responses made by any participant in either group. The rate of 'anticipations' (defined as responding $<100 \mathrm{~ms}$ after presentation of the target stimulus) was very low and did not differ between controls and BD patients ( $1.08 \pm 1.4$ vs $1.0 \pm 1.3$ respectively; $t=0.275$ $p=0.784)$. As a result anticipations were not analysed further. Mean RT and number of lapses was significantly greater ( $p=0.047$ and 0.050 respectively) in the 26 euthymic BD patients compared to the controls, though the effect sizes were a little smaller $(g=0.65(95 \% \mathrm{Cl}=0.13-1.17)$ and $g=0.53$ $(95 \% \mathrm{Cl}=0.02-1.05$ respectively) than for the comparison including all patients. However, the BD patients with normal sleep did not differ from controls on either metric, while the BD patients with abnormal sleep did (mean RT: $p<0.001 ; g=1.18,95 \% \mathrm{Cl}=0.64-1.72 ;$ lapses: $\mathrm{p}<0.001 ; \mathrm{g}=0.91$, $95 \% \mathrm{Cl}=0.39-1.43)$. This was also the case for the BD patients with abnormal sleep but who were euthymic (mean RT: $p=0.001 ; g=1.26,95 \% \mathrm{Cl}=0.54-1.98$; lapses: $\mathrm{p}=0.005 ; \mathrm{g}=1.04,95 \% \mathrm{Cl}=$ $0.33-1.75)$. Mean RTs are shown in the various subgroups in figure 1. The differences from controls were consistent across each of the three most common BD sleep phenotypes in this cohort $(p<0.001$ for the difference in mean RT versus controls for all three - see figure 1$)$. There were no significant correlations with age or NART IQ and so ANCOVA controlling for these variables was not performed.

Figure 1 near here

Ex-Gaussian analysis of PVT RTs found a significantly greater $m u(p<0.05 ; g=0.52,95 \% \mathrm{Cl}=0.08-$ $0.97)$ and tau $(\mathrm{p}<0.01, \mathrm{~g}=0.65,95 \% \mathrm{Cl}=0.20-1.10)$, though not sigma, in BD patients compared 
to controls. The increase in tau is consistent with increased IIV and the higher rate of lapses seen with the raw RT data. There were no significant differences from controls in either the euthymic BD sub-group nor the BD patients with normal sleep, in either mu, sigma or tau. However, the BD patients with abnormal sleep had significantly greater $m u(p<0.01 ; g=0.79,95 \% \mathrm{Cl}=0.27-1.30)$, sigma $(\mathrm{p}<0.005 ; \mathrm{g}=0.87,95 \% \mathrm{Cl}=0.34-1.39)$ and $\operatorname{tau}(\mathrm{p}<0.001 ; \mathrm{g}=0.85,95 \% \mathrm{Cl}=0.33-1.37)$. In the sub-group of BD patients with abnormal sleep who were euthymic, sigma and tau remained significantly greater than in the control participants with similar magnitude effect sizes. Ex-Gaussian distributions of the PVT RTs are shown in figure 2, where it can be seen that the three common abnormal sleep phenotypes had similar distributions. There were no significant correlations of age or NART-IQ in the control group and therefore ANCOVA controlling for these variables was not performed.

Figure 2 near here

\section{Attention Network Task (ANT).}

The ANT was completed by 35 controls and 44 BD patients. One control was omitted from the analysis as their mean RT and conflict RT were more than 3 times the interquartile range and they were considered an extreme outlier. Mean ANT RTs showed a similar pattern of effects to PVT RTs, with BD patients having significantly greater meant RTs than controls, euthymic and BD normal sleepers not differing from controls, but BD abnormal sleepers having significantly greater mean RTs than controls $(p=0.001 ; g=0.92,95 \% \mathrm{Cl}=0.40-1.45)$, including just those who were euthymic $(p=$ $0.002 ; \mathrm{g}=1.13,95 \% \mathrm{Cl}=0.41-1.85)$. Ex-Gaussian analysis also revealed an identical pattern of effects to that seen with the PVT RT.

The main purpose of the ANT is to assess the processing efficiency of the alerting, orientating and executive attentional networks (Fan, McCandliss, Sommer, Raz, \& Posner 2002). BD patients differed from control participants on the orientating $(\mathrm{p}<0.05 ; \mathrm{g}=0.49 ; 95 \% \mathrm{Cl}=0.04-0.95)$ and conflict $(p=0.005 ; g=0.68 ; 95 \% \mathrm{Cl}=0.22-1.14)$ RTs, the latter representing the executive network. 
In the comparison between controls and euthymic BD patients and BD patients with normal sleep, the orientating RTs remained significantly different, though the conflict RTs were not significantly different. However, the BD patients with abnormal sleep differed from controls on the conflict RT with a large effect size $(p<0.001 ; g=1.14 ; 95 \% \mathrm{Cl}=0.61-1.68)$ but with no difference in alerting or orientating RTs. The euthymic BD abnormal sleepers also differed from controls on the conflict RT ( $p$ $<0.01 ; \mathrm{g}=1.15 ; 95 \% \mathrm{Cl}=0.43-1.87)$ but not the other two RTs. This finding suggests that abnormal BD sleepers have an impaired executive attentional network compared to controls and that is not dependent on mood state. This impairment in executive attentional network was also evident in all three BD sleep phenotypes ( $p<0.01$ for all phenotypes).

There was no correlation between age or NART IQ and mean ANT RT, conflict or orientating RT and so no ANCOVA was performed.

\section{Digit symbol substitution test (DSST)}

The DSST was completed at the beginning and end of the cognitive test battery to check for evidence of fatigue. Performance on the second occasion was statistically significantly better, though with only a small effect size. Only data from the first DSST to be performed is reported here, though the findings are identical if using the second DSST data. The findings are shown in figure 3 . As can be seen, the pattern of effects in the various subgroups is very similar to that seen for the mean PVT RT shown in figure 1. As expected, BD patients made fewer correct responses than the controls $(p<$ $0.001 ; \mathrm{g}=-0.77 ; 95 \% \mathrm{Cl}=-1.22--0.32)$. This was also the case in the euthymic BD patients $(\mathrm{p}<0.05$; $\mathrm{g}=-0.60 ; 95 \% \mathrm{Cl}=-1.11--0.08)$. However, the BD patients with normal sleep did not differ from controls, while those with abnormal sleep did $(p<0.001 ; g=-0.98 ; 95 \% \mathrm{Cl}=-1.48--0.44)$, including just those who were euthymic $(p<0.01 ; g=-0.91 ; 95 \% \mathrm{Cl}=-1.61--0.21)$. The sub-groups of BD patients with the three main abnormal sleep phenotypes seen also all different from controls (all $p<$

0.05). There was a significant Pearson's correlations between age and DSST score in controls $(r(36)=$ $0.689, p<0.001)$ and patients $(r(46)=0.472, p=0.003)$. Age was therefore included as a covariate in 
ANCOVA analysis of all of the comparisons between BD patient subgroups and controls. All differences remained significant.

Figure 3 near here

TRAILS A and B.

One control participant was not included in this analysis as their TRAILS B-A score was greater than 3 times the interquartile range and was therefore deemed extreme outlier. There were no significant differences between controls and BD patients in performance on the TRAILS A or B, or the B minus A score. As a result, no subgroup analysis was performed.

\section{Digit span test}

There were no significant differences between controls and BD patients in performance on the digit span total scores. Correlation analysis however found a significant Pearson's correlation between NART-IQ and digit span score in controls $\left(r_{(36)}=0.484, p=0.003\right)$ and BD patients $\left(r_{(46)}=0.302\right.$, $p=0.042$ ). ANCOVA was therefore performed to control for the effects of IQ. This revealed a significant difference between controls and patients $\left(F_{(1,78)}=5.833, p=0.018\right)$, including just those who were euthymic $\left(F_{(1,58)}=4.159, p=0.046\right)$ but not those with normal sleep $\left(F_{(1,50)}=3.355\right.$, $p=0.073)$. However, patients with abnormal sleep did differ from controls $\left(F_{(1,60)}=5.165, p=0.027\right)$ although in this instance this was not seen in the abnormal sleepers who were euthymic $\left(F_{(1,43)}=\right.$ 2.717, $p=0.107)$.

\section{Newcastle Spatial Working Memory (NSWM) Test}

BD patients committed significantly more between search errors on the NSWM test than controls ( $p$ $<0.05 ; \mathrm{g}=0.023,95 \% \mathrm{Cl}=0.07-0.99)$. There was however a moderate correlation between age and between search errors in controls $\left(r_{(33)}=0.562, p=0.001\right)$ and a weak correlation in BD patients $\left(r_{(43)}=0.300, p=0.051\right)$. After controlling for age with ANCOVA, the differences between controls and BD patients was no longer significant. As a result, no further analysis was performed. 


\section{Verbal learning test.}

BD patients recalled significantly fewer words on the immediate recall of the verbal learning test $(p=$ $0.002 ; \mathrm{g}=-0.60,95 \% \mathrm{Cl}=-1.05--0.16)$. There was a significant Pearson's correlation between NARTIQ and immediate recall score in BD patients $\left(r_{(46)}=0.413, p=0.004\right)$ and a trend towards a significant correlation in controls after winsorisng a significant outlier $\left(r_{(36)}=.314, p=0.066\right)$. ANCOVA covarying for NART-IQ found a significant difference between BD patients and controls $\left(\mathrm{F}_{(1,78)}=13.999\right.$, $p<0.001)$, that was also seen for the euthymic $\left(F_{(1,58)}=4.551, p=0.037\right)$ but not normal sleeping $\left(F_{(1,48)}\right.$ $=3.681, p=0.061)$ subgroups. BD abnormal sleeps, however, did differ from controls $\left(F_{(1,60)}=12.806\right.$, $p=0.001)$ though not just those who were in euthymia $\left(F_{(1,43)}=1.982, p=0.166\right)$.

\section{Discussion}

This is the most comprehensive study to date to assess objectively defined abnormal sleep / circadian rhythm and cognition in BD patients compared to controls. In summary, across a wide range of cognitive assessments, there were the expected significant differences between BD patients as a group compared to age and sex matched controls. However these differences were almost entirely associated with those BD patients with objectively defined abnormal sleep. BD patients with normal sleep did not differ from controls on any cognitive measure with the exception of the ANT orientating network RTs. Given that no corrections were made for multiple comparisons, this could be a type I error. Conversely, BD patients with abnormal sleep differed from controls having longer PVT and mean ANT RTs, greater RT IIV, impaired executive attentional network function, fewer correct responses on the DSST, poorer digit span performance and worse verbal memory recall. While BD patients in our sample with abnormal sleep had lower mood than those with normal sleep (Bradley, Webb-Mitchell, Hazu, Slater, Middleton, Gallagher, McAllister-Williams, \& Anderson 2017), BD patients with abnormal sleep but who were euthymic, also differed from the controls on all of these cognitive measures except digit span and verbal memory. Given the relatively small sample 
size of BD patients with abnormal sleep but who were in euthymia, the lack of statistical significance in the difference in digit span and verbal memory may well be due to a lack of statistical power. While we therefore confirmed cognitive dysfunction in $\mathrm{BD}$, especially in measures of attention, rather than sleep abnormalities exacerbating such dysfunction, the cognitive abnormalities were entirely confined to BD patients with sleep abnormalities. As such, these findings are therefore consistent with sleep disturbance being the main driver of the cognitive abnormalities seen in BD.

\section{Limitations}

Weaknesses of the study include a lack of the gold standard measure of sleep using video polysomnography. However this was a field study and it was felt that studying patients with partial polysomnography in their own homes would increase compliance. The sample was opportunistic with a potential bias for over-representation of patients with BD and sleep disorders, while controls were screened out if they suffered significant sleep problems. A larger, more representative, sample size would have allowed greater sub group analysis of the differential effect of the different patterns of sleep and circadian rhythm. Future studies might include those with other psychiatric disorders or control subjects with primary sleep disorders to study the impact upon cognition.

Actigraphy assesses physical activity and hence is a surrogate marker of sleep. However, it has been the most widely used and published technique to assess sleep/wake patterns and circadian rhythm for many years (Melo et al. 2016). The American Academy of Sleep Medicine also recognise actigraphy as sufficient, alongside clinical evaluation, to make the diagnosis of circadian rhythm disorders (American Academy of Sleep Medicine 2014). In bipolar disorder, sleep variables derived from actigraphy have been shown to highly correlate with gold standard polysomnography (Kaplan et al. 2012). As a result, while the use of actigraphy is a limitation, we believe that it is a legitimate and pragmatic method for assessing sleep. 
The patients included in this study were not medication free and it is not possible to exclude the possibility that this influenced our findings. However, there was no difference in the rates of usage of different medications between the different bipolar subgroups with the exception of a significantly higher rate of hypnotic use in in long sleepers (42.9\%) compared to normal sleepers (6.3\%) (Fisher's exact test $p=0.031$ ). This finding and details of the medication used is provided in our previous publication (Bradley, Webb-Mitchell, Hazu, Slater, Middleton, Gallagher, McAllisterWilliams, \& Anderson 2017).

No correction was made for multiple statistical testing. However, it seems unlikely that our findings are type I errors for two main reasons. Firstly, there was consistency in the findings both between measures of the same cognitive domain (attention - PVT and ANT) and across domains. Secondly, if a conservative Bonferroni correction had been used the main bulk of our findings would have remained significant, including the difference between bipolar patients with abnormal sleep and controls (PVT RTs and lapses, ANT RT, DSST, verbal learning) and between euthymic bipolar patients with abnormal sleep and controls (PVT RTs and lapses, ANT RT). The findings that would not have remained significant are the DSST difference between controls and euthymic bipolar abnormal sleepers and controls and the findings with the digit span test. We did not apply a Bonferonni correction since we were not interested in whether patients and controls differed cognitively, but in which domain with a particular a priori focus on attention and psychomotor processing speed. Applying corrections for multiple comparisons would simply have increased the risk of type II errors. Rather we rely upon describing the statistical tests performed and the pattern of effects seen (Perneger 1998). For all contrasts we include estimates of effect size with accompanying $95 \% \mathrm{Cl}$ which are more informative to future research that the point estimates of significance which we agree are linked to sample size and therefore limited in their utility.

The study also had a number of strengths. These include a well characterised group and comprehensive measures of cognition, sleep and circadian rhythm across 21 days. The control group 
were carefully matched and a wide range of sleep disturbances was included to reflect real life clinical practice where many BD patients have a variety of reasons for disturbed sleep. We were fortunate enough to have a reasonable number of both normal and abnormal sleepers, and of euthymic patients in the latter group. This allowed for an examination of our hypotheses without the need for spuriously covarying for mood in comparisons between two groups (patients and controls) that differed on their mood ratings.

In conclusion we have found that many patients with BD have disrupted sleep and that this has a significant impact upon tests of cognition including in those patients in remission. This suggests that objective measures of sleep disturbance need to be taken into account in future studies of cognition in $\mathrm{BD}$ and that sleep may be a potential target for treatment of cognitive disturbance in BD.

\section{Acknowledgements and Financial Support}

We would like to thank all of the participants for taking part in the study. The study was sponsored by Northumberland, Tyne and Wear NHS Foundation Trust. Some funding to cover running costs was received from Eli Lilly as an unrestricted investigator initiated and led award. The design, conduct and analysis of the study was entirely under the control of the authors.

\section{Conflict of interest}

None.

\section{Ethical standards}

The authors assert that all procedures contributing to this work comply with the ethical standards of the relevant national and institutional committees on human experimentation and with the Helsinki Declaration of 1975, as revised in 2008. 


\section{Refernces}

American Academy of Sleep Medicine 2014. International classification of sleep disorders Darien, Ilinois, American Academy of Sleep Medicine.

American Psychiatric Association 1994. Diagnostic and Statistical Manual of Mental Disorders Fourth Edition (DSM-IV) Washington, DC, American Psychiatric Association.

Beck, A.T., Ward, C.H., Mendelson, M., Mock, J., \& Erbaugh, J. 1961. An inventory for measuring depression. Archives of General Psychiatry, 4, 561-571 available from:

http://www.sciencedirect.com/science/article/B6WVB-4F9MR491SB/2/ae36a31f5817e3647fa0ff86fab4a2ea

Bora, E. 2018. Neurocognitive features in clinical subgroups of bipolar disorder: A meta-analysis. Journal of Affective Disorders, 229, 125-134 available from: PM:29306692

Bourne, C., Aydemir, O., Balanza-Martinez, V., Bora, E., Brissos, S., Cavanagh, J.T., Clark, L., Cubukcuoglu, Z., Dias, V.V., Dittmann, S., Ferrier, I.N., Fleck, D.E., Frangou, S., Gallagher, P., Jones, L., Kieseppa, T., Martinez-Aran, A., Melle, I., Moore, P.B., Mur, M., Pfennig, A., Raust, A., Senturk, V., Simonsen, C., Smith, D.J., Bio, D.S., Soeiro-de-Souza, M.G., Stoddart, S.D., Sundet, K., Szoke, A., Thompson, J.M., Torrent, C., Zalla, T., Craddock, N., Andreassen, O.A., Leboyer, M., Vieta, E., Bauer, M., Worhunsky, P.D., Tzagarakis, C., Rogers, R.D., Geddes, J.R., \& Goodwin, G.M. 2013. Neuropsychological testing of cognitive impairment in euthymic bipolar disorder: an individual patient data meta-analysis. Acta Psychiatrica Scandinavica, 128, (3) 149-162 available from: PM:23617548

Bradley, A.J., Webb-Mitchell, R., Hazu, A., Slater, N., Middleton, B., Gallagher, P., McAllister-Williams, H., \& Anderson, K.N. 2017. Sleep and circadian rhythm disturbance in bipolar disorder. Psychological Medicine, 47, (9) 1678-1689 available from: PM:28173893

Bucks, R.S., Olaithe, M., \& Eastwood, P. 2013. Neurocognitive function in obstructive sleep apnoea: a meta-review. Respirology., 18, (1) 61-70 available from: PM:22913604

Buysse, D.J., Reynolds, C.F., III, Monk, T.H., Berman, S.R., \& Kupfer, D.J. 1989. The Pittsburgh Sleep Quality Index: a new instrument for psychiatric practice and research. Psychiatry Research, 28, (2) 193-213 available from: PM:2748771

Dinges, D.F. \& Powell, J.W. 1985. Microcomputer analyses of performance on a portable, simple visual RT task during sustained operations. Behavior Research Methods, 17, 652-655

Fan, J., McCandliss, B.D., Sommer, T., Raz, A., \& Posner, M.I. 2002. Testing the efficiency and independence of attentional networks. Journal of Cognitive Neuroscience, 14, (3) 340-347

Gallagher, P., Gray, J.M., Watson, S., Young, A.H., \& Ferrier, I.N. 2014. Neurocognitive functioning in bipolar depression: a component structure analysis. Psychological Medicine, 44, (5) 961-974 available from: PM:23800475

Gallagher, P., Nilsson, J., Finkelmeyer, A., Goshawk, M., Macritchie, K.A., Lloyd, A.J., Thompson, J.M., Porter, R.J., Young, A.H., Ferrier, I.N., McAllister-Williams, R.H., \& Watson, S. 2015. Neurocognitive intra-individual variability in mood disorders: effects on attentional response time distributions. Psychological Medicine, 45, (14) 2985-2997 available from: PM:26073667 
Giglio, L.M., Magalhaes, P.V., Kapczinski, N.S., Walz, J.C., \& Kapczinski, F. 2010. Functional impact of biological rhythm disturbance in bipolar disorder. J.Psychiatr.Res., 44, (4) 220-223 available from: PM:19758600

Hamilton, M. 1967. Development of a rating scale for primary depressive illness. British Journal of Social \& Clinical Psychology, 6, (4) 278-296

Harvey, A.G., Schmidt, D.A., Scarna, A., Semler, C.N., \& Goodwin, G.M. 2005. Sleep-related functioning in euthymic patients with bipolar disorder, patients with insomnia, and subjects without sleep problems. American Journal of Psychiatry, 162, (1) 50-57

Johns, M.W. 1991. A new method for measuring daytime sleepiness: the Epworth sleepiness scale. Sleep, 14, (6) 540-545 available from: PM:1798888

Kanady, J.C., Soehner, A.M., Klein, A.B., \& Harvey, A.G. 2017. The association between insomniarelated sleep disruptions and cognitive dysfunction during the inter-episode phase of bipolar disorder. J.Psychiatr.Res., 88, 80-88 available from: PM:28088728

Kaplan, K.A., Gruber, J., Eidelman, P., Talbot, L.S., \& Harvey, A.G. 2011. Hypersomnia in inter-episode bipolar disorder: does it have prognostic significance? Journal of Affective Disorders, 132, (3) 438444 available from: PM:21489637

Kaplan, K.A., Talbot, L.S., Gruber, J., \& Harvey, A.G. 2012. Evaluating sleep in bipolar disorder: comparison between actigraphy, polysomnography, and sleep diary. Bipolar.Disord., 14, (8) 870-879 available from: PM:23167935

Keller, M.B. 2003. Past, present, and future directions for defining optimal treatment outcome in depression: remission and beyond. Journal of the American Medical Association, 289, (23) $3152-$ 3160 available from: PM:12813121

Kushida, C.A., Littner, M.R., Morgenthaler, T., Alessi, C.A., Bailey, D., Coleman, J., Jr., Friedman, L., Hirshkowitz, M., Kapen, S., Kramer, M., Lee-Chiong, T., Loube, D.L., Owens, J., Pancer, J.P., \& Wise, M. 2005. Practice parameters for the indications for polysomnography and related procedures: an update for 2005. Sleep, 28, (4) 499-521 available from: PM:16171294

Lacouture, Y. \& Cousineau, D. 2008. How to use MATLAB to fit the ex-Gaussian and other probability functions to a distribution of response times. Methods for Psychology, 4, 35-45

Lezak, M.D., Howieson, D.B., \& Loring, D.W. 2004. Neuropsychological assessment Oxford, UK, Oxford University Press.

Lim, J. \& Dinges, D.F. 2008. Sleep deprivation and vigilant attention. Annals of the New York Academy of Sciences, 1129, 305-322 available from: PM:18591490

Melo, M.C., Garcia, R.F., Linhares Neto, V.B., Sa, M.B., de Mesquita, L.M., de Araujo, C.F., \& de Bruin, V.M. 2016. Sleep and circadian alterations in people at risk for bipolar disorder: A systematic review. J.Psychiatr.Res., 83, 211-219 available from: PM:27661417

Nelson, H.E. 1982. The National Adult Reading Test (NART): Test Manual Windsor, England, NFER Publishing Co..

Pariante, C.M., Alhaj, H.A., Arulnathan, V.E., Gallager, P., Hanson, A., Massey, A.E., \& McAllisterWilliams, R.H. 2012. Central glucocorticoid receptor-medicated effects of the antidepressant 
citalopram in humans: A study using EEG and cognitive testing. Psychoneuroendocrinology, 37, (5) 618-628

Perneger, T.V. 1998. What's wrong with Bonferroni adjustments. British Medical Journal, 316, (7139) 1236-1238 available from: PM:9553006

Ratcliff, R. 1979. Group reaction time distributions and an analysis of distribution statistics.

Psychological Bulletin, 86, (3) 446-461 available from: PM:451109

Robinson, L.J., Thompson, J.M., Gallagher, P., Goswami, U., Young, A.H., Ferrier, I.N., \& Moore, P.B. 2006. A meta-analysis of cognitive deficits in euthymic patients with bipolar disorder. Journal of Affective Disorders, 93, (1-3) 105-115 available from: PM:16677713

Samame, C., Martino, D.J., \& Strejilevich, S.A. 2014. Longitudinal course of cognitive deficits in bipolar disorder: a meta-analytic study. Journal of Affective Disorders, 164, 130-138 available from: PM:24856566

Schmiedek, F., Oberauer, K., Wilhelm, O., Suss, H.M., \& Wittmann, W.W. 2007. Individual differences in components of reaction time distributions and their relations to working memory and intelligence. Journal of Experimental Psychology: General, 136, (3) 414-429 available from: PM:17696691

Sheehan, D.V., Lecrubier, Y., Sheehan, K.H., Amorim, P., Janavs, J., Weiller, E., Hergueta, T., Baker, R., \& Dunbar, G.C. 1998. The Mini-International Neuropsychiatric Interview (M.I.N.I.): the development and validation of a structured diagnostic psychiatric interview for DSM-IV and ICD-10. Journal of Clinical Psychiatry, 59 Suppl 20, 22-33 available from: PM:9881538

Soreca, I., Buttenfield, J.A., Hall, M.H., \& Kupfer, D.J. 2015. Screening for obstructive sleep apnea in patients with bipolar I disorder: comparison between subjective and objective measures. Bipolar.Disord., 17, (3) 345-348 available from: PM:25187351

Thompson, J.M., Gallagher, P., Hughes, J.H., Watson, S., Gray, J.M., Ferrier, I.N., \& Young, A.H. 2005. Neurocognitive impairment in euthymic patients with bipolar affective disorder. British Journal of Psychiatry, 186, 32-40

van Hees, V.T., Sabia, S., Anderson, K.N., Denton, S.J., Oliver, J., Catt, M., Abell, J.G., Kivimaki, M., Trenell, M.I., \& Singh-Manoux, A. 2015. A Novel, Open Access Method to Assess Sleep Duration Using a Wrist-Worn Accelerometer. PLoS.One., 10, (11) e0142533 available from: PM:26569414

Van Someren, E.J., Swaab, D.F., Colenda, C.C., Cohen, W., McCall, W.V., \& Rosenquist, P.B. 1999. Bright light therapy: improved sensitivity to its effects on rest-activity rhythms in Alzheimer patients by application of nonparametric methods. Chronobiol.Int., 16, (4) 505-518 available from: PM:10442243

Walters, A.S., LeBrocq, C., Dhar, A., Hening, W., Rosen, R., Allen, R.P., \& Trenkwalder, C. 2003. Validation of the International Restless Legs Syndrome Study Group rating scale for restless legs syndrome. Sleep Med., 4, (2) 121-132 available from: PM:14592342

Wechsler, D. 1981. WAIS-R manual, Wechsler Adult Intelligence Scale-Revised Cleveland, OH, Psychological Corp.

Williams, J.B., Kobak, K.A., Bech, P., Engelhardt, N., Evans, K., Lipsitz, J., Olin, J., Pearson, J., \& Kalali, A. 2008. The GRID-HAMD: standardization of the Hamilton Depression Rating Scale. International Clinical Psychopharmacology, 23, (3) 120-129 
Young, R.C., Biggs, J.T., Ziegler, V.E., \& Meyer, D.A. 1978. A rating scale for mania: reliability, validity and sensitivity. Br.J.Psychiatry, 133, 429-435 available from: PM:728692 


\section{Figure Legends}

Figure 1. PVT mean response times

Response times (mean with the error bars representing the SEM) on the PVT task for the healthy controls $(n=36)$ and BD patients. The total BD group is shown $(n=45)$ along with subsets of the BD patients including those who were euthymic $(n=26)$; those with normal sleep $(n=16)$; those with abnormal sleep (27); those with abnormal sleep who were euthymic $(n=11)$; those BD patients with long sleep $(n=13)$; those BD patients with CRSD $(n=12)$ and those BD patients with OSA $(n=12){ }^{*}=p<0.05 ;{ }^{* * *}=p<0.001$ compared to controls.

$\mathrm{BD}=$ bipolar disorder; $\mathrm{CRSD}=$ circadian rhythm sleep disorder; OSA = Obstructive sleep apnoea; PVT = Psychomotor vigilence task; SEM = Standard error of the mean.

\section{Figure 2. PVT response time distributions}

Response time distributions plotted for controls ( $n=36), B D$ patients with normal sleep $(n=16)$ and BD patients with abnormal sleep $(n=27)$. Data plotted in Prism v7.01, 2016 (GraphPad Software Inc, La Jolla, CA) with a smoothed curve based on a rolling average utilising 4 data points on each side of each data point.

$\mathrm{BD}=$ bipolar disorder; PVT = Psychomotor vigilence task.

\section{Figure 3. DSST performance}

Number of correct responses made in 90 seconds (mean with the error bars representing the SEM) for the healthy controls $(n=36)$ and BD patients. The total BD group is shown $(n=46)$ along with sub-sets of the BD patients including those who were euthymic $(n=26)$; those with normal sleep $(n=16)$; those with abnormal sleep (28) and those with abnormal sleep who were euthymic $(n=11)$ those BD patients with long sleep $(n=14)$; those BD patients with CRSD $(n=12)$ and those BD patients with OSA $(n=12) .{ }^{*}=p<0.05 ;{ }^{*}=p<$ $0.01 ;{ }^{* * *}=\mathrm{p}<0.001$ compared to controls.

$\mathrm{BD}=$ bipolar disorder; CRSD = circadian rhythm sleep disorder; DSST = Digit symbol substitution test; OSA = Obstructive sleep apnoea; SEM = Standard error of the mean. 
Table 1: General characteristics of study participants at baseline (day 1)

\begin{tabular}{|c|c|c|c|c|c|}
\hline \multirow[b]{2}{*}{ Characteristic } & \multirow[b]{2}{*}{ Controls $(n=36)$} & \multirow[b]{2}{*}{ Bipolar Disorder $(n=46)$} & \multicolumn{3}{|c|}{ Statistical test } \\
\hline & & & $x^{2}(d f)$ & Mann Whitney U & P value \\
\hline Female gender, $\mathrm{n}(\%)$ & $25(69.4 \%)$ & $31(67.4 \%)$ & $0.039(1)$ & & 0.843 \\
\hline Age years: mean (SD) & $42.8(11.9)$ & $46.8(11.1)$ & & 670.0 & 0.140 \\
\hline NART (SD) & $116.5(8.0)$ & $119.5(7.2)$ & & 632.5 & 0.099 \\
\hline (Range) & $(97-128)$ & $(97-131)$ & & & \\
\hline Years in full time education (SD) & $15.3(3.0)$ & $15.8(3.5)$ & & 755.5 & 0.495 \\
\hline (Range) & $(11-22)$ & $(10-24)$ & & & \\
\hline BMI mean: $\mathrm{kg} / \mathrm{m}^{2}$ (SD) & $25.1(4.4)$ & $30.0(6.7)$ & & 427.0 & $<0.001$ \\
\hline (Range) & $(19.5-36.4)$ & $(21.0-52.0)$ & & & \\
\hline $\mathrm{BMI} \geq 30 \mathrm{~kg} / \mathrm{m}^{2}, \mathrm{n}(\%)$ & $6(17.1 \%)$ & $19(41.3 \%)$ & $5.438(1)$ & & 0.02 \\
\hline Smoker, n (\%) & $3(8.3 \%)$ & $6(13 \%)$ & $0.459(1)$ & & 0.724 \\
\hline Mean alcohol units per week & $8.7(7.7)$ & $4.9(9.8)$ & & 474.5 & 0.001 \\
\hline (range) & $(0-30)$ & $(0-50)$ & & & \\
\hline HAMD-17 mean (SD) & $0.3(0.6)$ & $9.1(7.2)$ & & 42.0 & $<0.001$ \\
\hline (Range) & $(0-2)$ & $(0-35)$ & & & \\
\hline (Range) & $(0-8)$ & $(0-49)$ & & & \\
\hline Remission (BDI $\leq 8) n(\%)$ & $36(100 \%)$ & $24(52.2 \%)$ & & & \\
\hline YMRS mean (SD) & $0.1(0.4)$ & $0.9(2.2)$ & & 728.0 & 0.128 \\
\hline (Range) & $(0-2)$ & $(0-10)$ & & & \\
\hline PSQI Global score mean (SD) & $2.3(1.2)$ & $8.6(4.6)$ & & 125.0 & $<0.001$ \\
\hline (Range) & $(0-4)$ & $(1-18)$ & & & \\
\hline PSQI > 5 n (\%) & $0(100 \%)$ & $30(65.2 \%)$ & & & \\
\hline ESS mean (SD) & $3.8(2.4)$ & $6.2(4.9)$ & & 575.5 & 0.018 \\
\hline (Range) & $(0-9)$ & $(0-21)$ & & & \\
\hline$E S S \geq 10$ & $0(0 \%)$ & $9(19.6 \%)$ & & & \\
\hline
\end{tabular}


Table 2: Comparison of characteristics of participants with bipolar disorder (BD) in different subgroups

\begin{tabular}{|c|c|c|c|c|c|}
\hline Characteristic & $\begin{array}{l}\text { Euthymic BD } \\
\text { Patients }(n=24)\end{array}$ & $\begin{array}{l}\text { Non-Euthymic BD } \\
\text { Patients }(n=22)\end{array}$ & $\begin{array}{l}\text { BD with normal } \\
\text { sleep }(n=16)\end{array}$ & $\begin{array}{l}\text { BD with abnormal } \\
\text { sleep }(n=28)\end{array}$ & $\begin{array}{l}\text { Euthymic BD with } \\
\text { abnormal sleep } \\
(n=11)\end{array}$ \\
\hline Female gender, $\mathrm{n}(\%)$ & $17(70.8)$ & $14(63.6)$ & $12(75.0)$ & 19 (67.9) & $8(72.7)$ \\
\hline Age years: mean (SD) & $47.1(11.4)$ & $46.1(11.0)$ & $44.1(10.3)$ & $49.2(10.8)$ & $50.2(12.1)$ \\
\hline NART (SD) & $119.9(5.9)$ & $119.0(8.5)$ & $120.4(7.1)$ & $119.4(6.7)$ & $118.5(4.2)$ \\
\hline BMI mean: $\mathrm{kg} / \mathrm{m}^{2}$ (SD) & $29.8(7.1)$ & $30.1(6.4)$ & $28.3(4.2)$ & $31.0(7.8)$ & $32.2(8.9)$ \\
\hline HAMD-17 mean (SD) & $4.5(2.8)$ & $14.1(7.1)$ & $5.8(5.2)$ & $10.1(7.2)$ & $5.3(2.2)$ \\
\hline YMRS mean (SD) & $1.1(2.6)$ & $0.6(1.8)$ & $1.5(3.1)$ & $0.5(1.6)$ & $0.2(0.4)$ \\
\hline PSQI Global score mean (SD) & $6.4(3.7)$ & $11.0(4.4)$ & $6.6(4.2)$ & $9.9(4.6)$ & $7.9(4.3)$ \\
\hline ESS mean (SD) & $5.5(4.4)$ & $7.1(5.3)$ & $5.4(3.6)$ & $7.1(5.4)$ & $6.8(5.7)$ \\
\hline
\end{tabular}




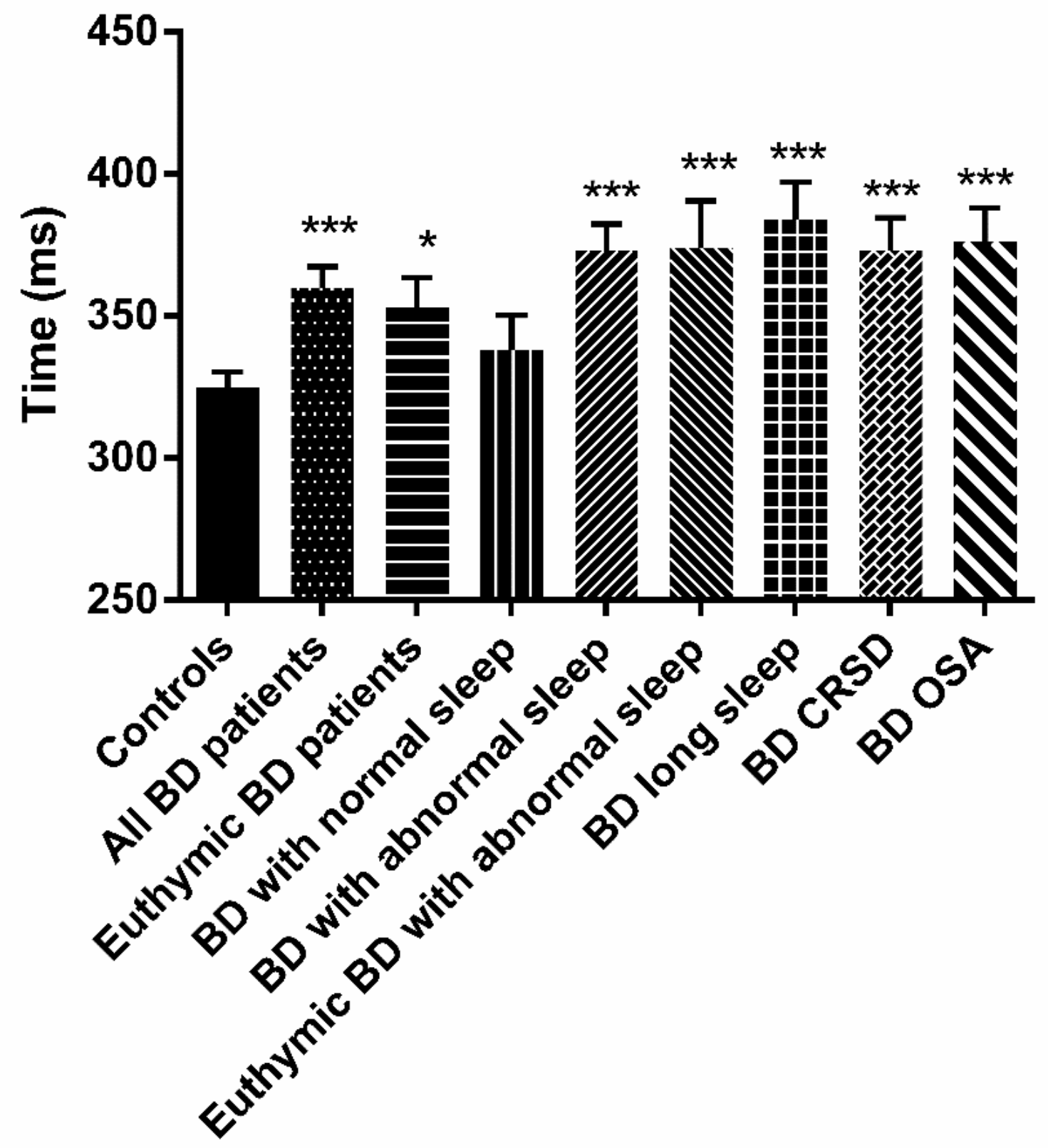

Figure 1. PVT all 
Figure 2

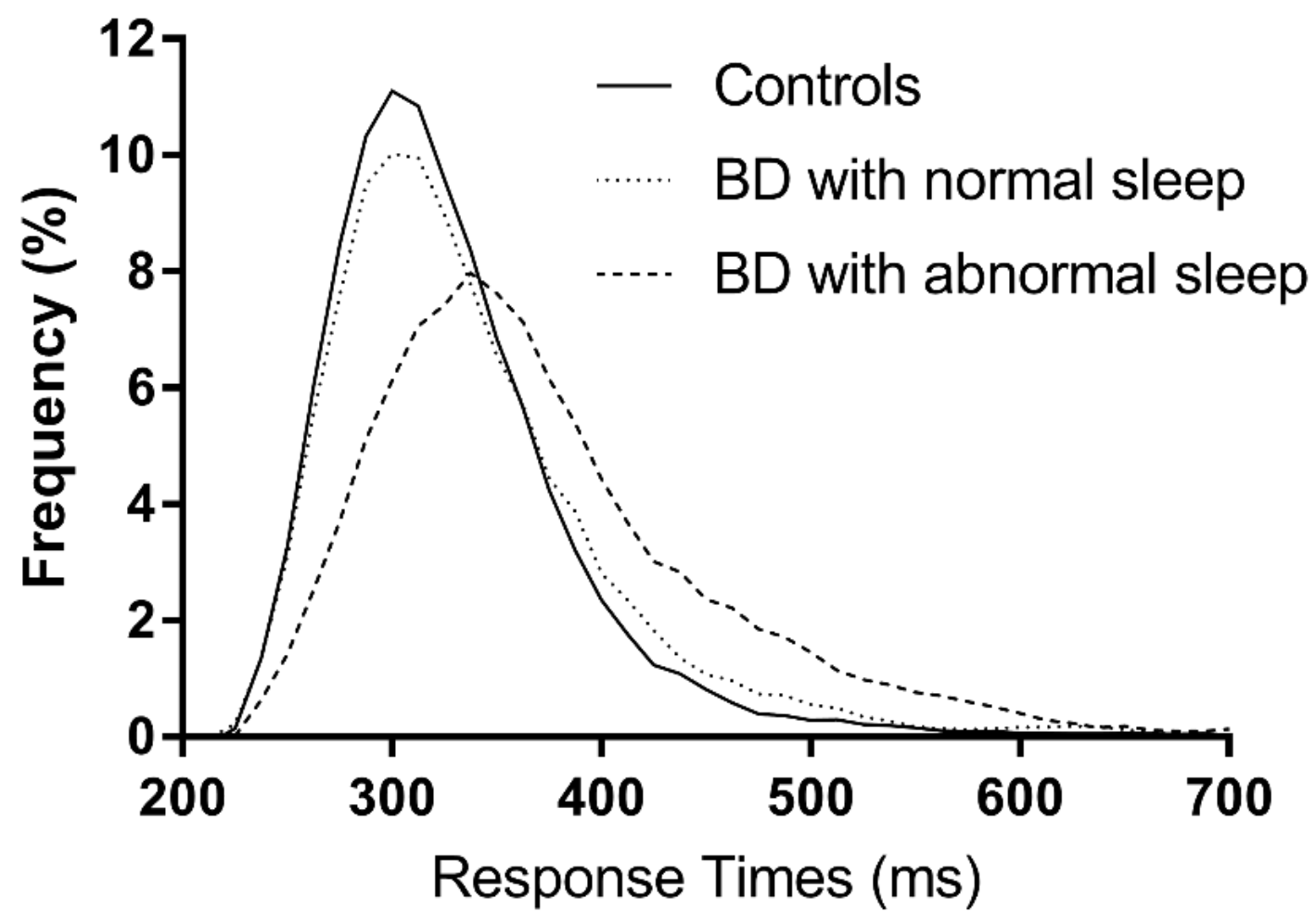


Figure 3. DSST all

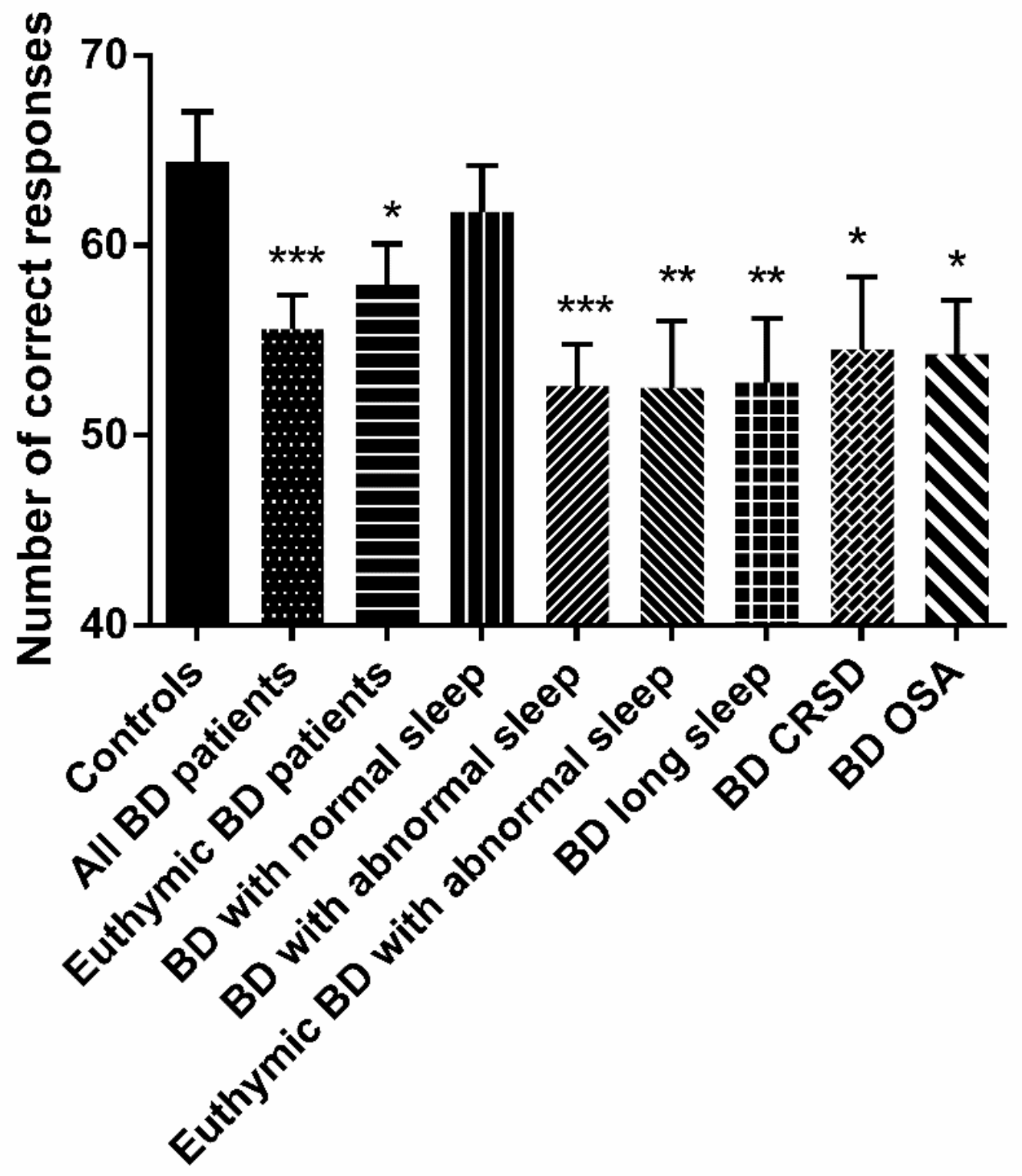

